\title{
Conservative treatment for advanced T3-T4 laryngeal cancer: meta-analysis of key oncological outcomes
}

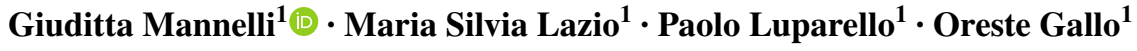

Received: 7 September 2017 / Accepted: 2 November 2017

(C) Springer-Verlag GmbH Germany, part of Springer Nature 2017

\begin{abstract}
Goal Controversies exist regarding the treatment of advanced laryngeal carcinomas. The purpose of this systematic review was to evaluate the oncologic outcomes of both transoral laser and open partial laryngectomies for advanced (T3-T4) squamous cell laryngeal cancers management.

Introduction A systematic review of literature was led searching for articles mentioning the following terms: advanced (T3-T4) laryngeal cancer AND laser; AND open partial laryngectomy; AND transoral laser microsurgery; AND cordectomy; AND conservative surgery; AND tracheohyoidopexy or tracheohyoidoepiglottopexy; AND supratracheal partial laryngectomy; AND supracricoid partial laryngectomy; AND cricohyoidopexy or cricohyoidoepiglottopexy. Then a quantitative analysis was carried on papers published after 1980 .

Discussion The search identified 110 publications, and a total of 21 articles satisfied inclusion criteria and were selected for quantitative synthesis. 10 out of 21 studies had a good quality score, 10 were fair and only one rated a poor score. The pooled disease-free survival (DFS) was $79 \%$ (95\% CI 74-85), and pooled overall survival (OS) was $71 \%$ (95\% CI 64-78) at 5 years from all 1921 patients included in the study, with significant heterogeneity $\left(I^{2}=89.7 \%\right.$ and $\left.I^{2}=90.4 \%\right)$, respectively. Significant heterogeneity value ( $p=0.118$ ) was seen by comparing transoral laser and open partial laryngectomies in terms of DFS.
\end{abstract}

Giuditta Mannelli

mannelli.giuditta@gmail.com

1 Clinic of Otorhinolaryngology, Head and Neck Surgery, Department of Translational Surgery and Medicine, University of Florence, Largo Brambilla 3, 50134 Firenze, Italy
Conclusion The two surgical techniques are both valid conservative surgical options for advanced laryngeal cancer treatment.

Keywords Systematic review - Meta-analysis - Advanced laryngeal carcinoma $\cdot$ Surgical conservative treatment .

Oncological outcomes.

\section{Introduction}

Laryngeal cancer is one of the most common cancer of the upper aerodigestive tract and the second among head and neck cancers after skin malignancies. Most of laryngeal neoplasms show squamous cell carcinoma (SCC) histology, accounting for $85-95 \%$ of laryngeal malignant tumours whose incidence is approximately $11,000-13,000$ of cases annually in the USA [1].

Advanced T stage (T3-T4) laryngeal cancer, encompass a heterogeneous variety of different lesions, each characterised by distinct clinical presentation, biological behaviours and prognosis. Thus, neoplastic pathways of tumour spread, should be carefully considered during therapeutic planning [2-4].

Currently, different modalities of treatment are available for all subtypes of T3 and T4 laryngeal tumours and, although oncologic outcome does remain the first goal, preservation of laryngeal functions and quality of life has progressively gained an important role heavily influencing the decision-making process of surgeons [5]. Since 1871, when Van Luschka introduced an accurate anatomical description of laryngeal framework by setting the body for future laryngeal surgery [6], over the last decades, the evolution of the treatment of laryngeal cancer has passed through different attitudes and aims, 
and in this scenario total laryngectomy (TL) has been the mainstay of treatment for decades. Afterwards, voice preservation has gained a crucial role in treatment planning and patients' expectations, consequently, patients have progressively begun to undergo surgical and nonsurgical preserving therapies [7]. The increasing accuracy of diagnostic techniques have gradually ensured a better patient selection, thus allowing the introduction of conservative surgery procedures in clinical practice. In fact, the proportion of patients receiving TL has declined from $74 \%$ in 1999 to $26 \%$ in 2007 [8] and open partial laryngectomy (OPL) together with transoral laser microsurgery (TLM), have been progressively introduced for the treatment of intermediate and advanced laryngeal tumours. Since the 1970s' when Labayle and Piquet introduced supracricoid partial laryngectomy for glottic cancer treatment $[9,10]$ and Steiner commenced TLM for laryngeal carcinomas in 1979 [11], laryngeal preservation procedures have paved the pathway for the contemporary management of laryngeal cancer. However, whereas chemoradiation therapy protocols have a tough scientific basis [12-14], indications for conservative surgery still wait for full validation.

Many publications, indeed, confirming the oncological and functional benefits of both OPL and TLM exist, and they show that both surgical procedures offer similar local control rates and overall survival to total laryngectomy for advanced laryngeal cancer treatment $[15,16]$, by ensuring fair post treatment organ functions when appropriate patients selection has been performed. In addition, consequently to the increasing use of chemotherapy with concurrent radiotherapy organ preservation strategies to treat advanced laryngeal cancer, OPLs have established an important and oncologically reliable role in the management of recurrent radio-resistant laryngeal carcinoma $[17,18]$.

In this respect, treatment plan selection might respect patients' needs, by aiming and demanding not only oncological but also functional results, in accordance with surgeons' skills and knowledge.

Given this background, we performed a systematic review of English language literature in an attempt to more robustly establish the local control and overall survival in conservation laryngeal surgery for advanced $\mathrm{T}$ stage cancers. The objective of this review and meta-analysis was to synthesise the oncological outcomes following laryngeal surgical preservation protocol, including both open partial and transoral laser procedures, performed as primary therapeutic options for advanced T3 and T4 laryngeal squamous cell carcinoma through the analysis of the latest reports in laryngeal cancer management published in the past 35 years, to give a full view about the most reliable therapeutic options for this type of patients.

\section{Materials and methods}

The systematic review was performed using independently developed search strategies in literature review methodology, and it was written in accordance with PRISMA Statement (http://www.prisma-statement.org) [19], to guarantee a scientific strategy of research to limit bias by a systematic assembly, critical appraisal and synthesis of all the most relevant studies published on this topic [20,21].

The databases interrogated included PubMed Clinical Queries http://www.ncbi.nlm.nih.gov. Reference lists from identified articles were searched and cross-referenced to identify additional relevant articles, and national experts in the field were contacted to identify unpublished data.

The search terms included the following various combinations to maximise the yield: advanced (T3-T4) laryngeal cancer AND laser; AND open partial laryngectomy (OPL); AND transoral laser microsurgery (TLM); AND cordectomy; AND conservative surgery; AND tracheohyoidopexy (THIP) or tracheohyoidoepiglotto-pexy (THIEP); AND supratracheal partial laryngectomy (STPL); AND supracricoid partial laryngectomy (SCPL) ; AND cricohyoidopexy (CHP) or cricohyoidoepiglotto-pexy (CHEP).

The search was performed for the first time on January 2016 and was set to automatically update periodically until December 2016.

First, duplicates were removed electronically. Then abstracts were reviewed to exclude obviously irrelevant articles. Non-English language papers and duplicates were excluded. Experimental studies and papers dealing with pathologies other than advanced lesions of the larynx were excluded.

The inclusion criteria were set a priori and deliberately kept wide to encompass as many articles as possible without compromising the validity of the results, and they included articles: (1) published from 1980 onwards, (2) reporting published series of $>10$ patients underwent partial laryngeal procedures with a minimum of 5 years follow up, (3) about conservation laryngeal procedures excluding total laryngectomy, (4) reporting data distinguishes results of partial laryngeal procedures divided into: TLM, CHP, CHEP, THIP, THIEP, SCL, HPL, Cordectomy, (5) with a clear description of TNM tumour stage and treatment selection criteria for primary laryngeal SCC, and (6) clear description of local control rate at 60 months.

The search excluded articles published before 1980 because the surgical repertoire until the 1960s was primarily restricted to TL as therapeutic surgical option for advanced laryngeal cancer and because reporting outcomes on relatively small case series with highly variable local control rates and functional outcomes. Furthermore, SCPL, although described much earlier, became more popular and common in clinical practice since the 1970s. Consequently, 
we considered articles published starting from the 1980s onwards to allow time for the flattening of the learning curve, together with the entrance in the surgical practice of more technological procedures, such as TLM for supraglottic lesions, which obviously belong to the latest years of our analysis.

We filtered the studies to ensure that only data from centres that had published on at least 10 patients were included in the review; this was done as a quality assurance measure as there are several case series in the literature, which have published the results of small numbers of cases spanning several years. Non-English language papers and duplicates were excluded.

All data were independently extracted by two authors and quality assessed. Eligibility for inclusion was separately assessed and when in doubt discussed and decided by consensus.

We chose overall survival (OS) and local control rate (LC) at 60 months as the primary measure of oncological outcome. Possible bias including age, existing co-morbidity, access and availability to treatment for other medical conditions and incidence of second primary tumours. Similarly disease-free survival (DFS) will depend on the incidence of regional and distant metastasis.

Abstracts were analysed to identify papers that fulfilled inclusion criteria and a first qualitative and descriptive review-analysis of selected articles was carried on; whilst, exclusively, publications clearly describing their aim and objectives, their inclusion and exclusion criteria, with clear or detachable statistical data, reporting adequate survival interval at 60 months from conservative surgical primary treatment, and well describing the surgical techniques and post-operative complications, were included in our metaanalysis. For articles not reporting raw data, letters were sent to the corresponding authors requesting them, otherwise they were excluded from the quantitative analysis. Raw data from the meta-analysis were entered into the appropriate contingency tables to allow calculation of OS and DFS, Younden's index, for each surgical technique: TLM, CHP, CHEP, THIP, THIEP, SCL, HPL, Cordectomy.
Given the variable reporting practice, the use of outcome measures that cannot be directly compared and the need for different search strategy, we did not include functional outcome in this meta-analysis as a criterion for inclusion.

\section{Study characteristics and quality assessment}

All included papers were graded using the NICE scoring scale for retrospective case series (Available at: http://www. nice.org.uk/nicemedia/pdf/Appendix_04_qualityofcase_ series_form_preop.pdf). This is a scoring scale with eight items, with each item scoring zero or one based on the study methods (Yes $=1 ; \mathrm{No}=0$ ). Scores of $\geq 6$ are considered to indicate a good quality study, scores between four and five as fair and those studies with a score of three are treated as poor quality (Table 1).

\section{Statistical analysis}

Fisher's exact test was used for statistical analysis of categorical data for the descriptive review, and a value of $p<0.05$ was considered significant.

The pooled estimate of each statistic was calculated after Freeman-Tukey double arcsine transformation to stabilise the variances. A random effect model was specified, using the method of DerSimonian and Laird, with the estimate of heterogeneity being taken from the inverse-variance fixedeffect model. Heterogeneity is also quantified using the I-squared measure.

Heterogeneity (or absence of homogeneity) of the results between the studies is assessed graphically by forest plots and statistically using the quantity $I^{2}$ that describes the percentage of total variation across studies that is attributable to heterogeneity rather than chance [22,23]. This is a measure of heterogeneity between the studies and ranges from 0 to $100 \%$; high figures indicate greater heterogeneity in the data.

When studies have low heterogeneity (pragmatically, $I^{2}<25 \%$ ), the differences between reported outcomes can be explained simply by the observed natural differences between patients. In this case, we can consider that all
Table 1 Quality assessment for case series
Quality assessment for case series

Case series collected in more than 1 center (i.e. a multicentric study) Is the hypothesis/aim/objective of the study clearly described?

Are the inclusion and exclusion criteria (case definition) celarly reported? Is there a clear definition of the outcomes reported?

Were data collected prospectively?

Is there an explicit statement that patients were recruited consecutively?

Are the main findings of the study clearly described?

Are outcomes stratified (i.e. by disease stage, abnormal test results, patient characteristics)? 
patients are part of the same larger pool. A fixed-effects meta-analysis is appropriate in which each patient is given approximately equal weight. However, with high heterogeneity, the studies differ by more than can be explained by intra-patients effects. This implies that there were differences in the patients studies, in the treatment interventions, or in the outcome measures. In this case, a random-effects meta-analysis is appropriate in which each study is given more equal weight.

All analyses were performed using STATA version 13 (StataCorp. 2013. Stata Statistical Software: Release 13. College Station, TX: StataCorp LP).

\section{Results}

The search strategy identified 110 articles in Medline from 1980 to the search date. They were selected, imported into Endnote, and the duplicates were removed. The removal of duplicates, non-English language works and of articles about other items rather than primary conservative surgical treatment for advanced laryngeal cancer yielded a total of 71 publications. 25 studies out of 71 were excluded from our analysis because reporting report, including letters or reviews, or not including advanced $\mathrm{T}$ stage laryngeal cancer but focusing only and general tumour stage. Therefore, 46 articles were considered for the qualitative systematic review whilst, only 21 out of 46, reporting on the outcomes of 1918 patients, were included in the meta-analysis.

The various stages of systematically assessing the abstracts and reasons for exclusion from the review are described in Fig. 1.

As for their quality, as assessed by the quality assessment criteria outlined above, 10 papers scored six or seven, 10 papers scored four or five and one paper scored three.

Of the included patients, 1448 and 470 patients were reported to have T3 and T4 tumour respectively, accordingly to the pathological AJCC classification [2].

The surgical procedures described in the 21 papers, included: transoral laser microsurgery (TLM), cricohyoidoepiglotto-pexy (CHEP), cricohyoidopexy (CHP), tracheohyoidoepiglotto-pexy (THIEP), tracheoepiglotto-pexy (THIP); supra tracheal partial laryngectomy (STLP), supracricoid partial laryngectomy (SCPL), horizontal partial laryngectomy (HPL), as reported in Table 2.

Adjuvant radiotherapy (RT) was not systematically administered by authors to every patient to avoid its potential collateral effects on their functional outcomes [31, 39]. The presence of positive resection margins after open partial surgery or patients with persistent tumour after transoral margins re-resection, large extra-laryngeal tumour extent, pathological report with presence of perineurial invasion and angioembolization, multiple positive lymph nodes and/or neck nodes with extra capsular spread (ECS) of the disease, represented the main conditions for adjuvant RT treatment indication.

Only three out of the 21 articles did not well specify indications and sites of adjuvant treatment [7, 34, 37]; on the other hand, the remaining 18 papers reported reasons and sites of post-operative RT. Table 2 summarises the number of patients who underwent adjuvant RT, where primary tumour and neck sites are reported separately.

Accordingly to our inclusion criteria, the follow-up duration time ranged from 12 to 120 months, with a median follow-up of 60 months.

The pooled OS (overall survival) was $71 \%$ (95\% CI 64-78). Heterogeneity tests revealed significant heterogeneity $\left(I^{2}=90.4 \%\right)$ which is to be expected given the wide spectrum of operations, outcomes and follow-up times reported. The lowest OS rate was $49.80 \%$ [32], with two studies reporting 94 and $93.80 \%$ OS rates $[34,42]$. The forest plot is shown in Fig. 2.

The pooled DFS (disease-free survival) was 79\% (95\% CI $74-85)$ with significant heterogeneity $\left(I^{2}=89.7 \%\right)$. The lowest DFS rate was $52.54 \%$ [32], with other two studies both reporting $93.75 \%$ of DFS [33, 42] and one study showing no recurrence event [35]. The forest plot is shown in Fig. 3.

Figures 4, 5 show forest forest plot by technique for OS and DFS, respectively. Here we divided surgical procedures into two different groups: (1) CLOSED techniques-TLM; vs. (2) OPEN techniques, including CHEP, CHP, THIEP, THIP, STLP, SCPL and HPL.

The pooled OS was the same in the two subgroups (heterogeneity between groups, $p$ value of 0.518 ) with 69\% (95\% CI 59-79) pooled OS rate in the closed technique group and 73\% (95\% CI 66-81) pooled OR rate in the open technique group. The pooled DFS was quite different in the two subgroups (heterogeneity between groups, $\mathrm{p}$ value of 0.118 ) with 76\% (95\% CI 69-83) pooled DSS rate in the closed technique group and $83 \%$ (95\% CI 78-89) pooled DFS rate in the open technique group.

\section{Discussion}

Despite its efficacy as an oncologic procedure, total laryngectomy (TL) implies complete loss of the larynx and represents a devastating event with significant subsequent diminution of quality of life for many individuals. Modern voice restoration procedures, have greatly improved the quality of life after TL but, since the 1980s it became apparent that in selected cases removal of the entire larynx was not necessary. In fact, conservation surgery complies with oncologic surgical principles and it has been shown that long-term survival rates are comparable to TL on well selected same advanced laryngeal lesions. In this respect, 


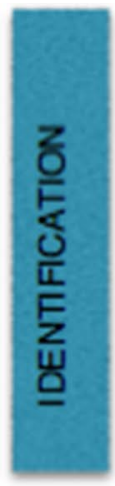

Systematic review in January 2016:

search through MEDLINE
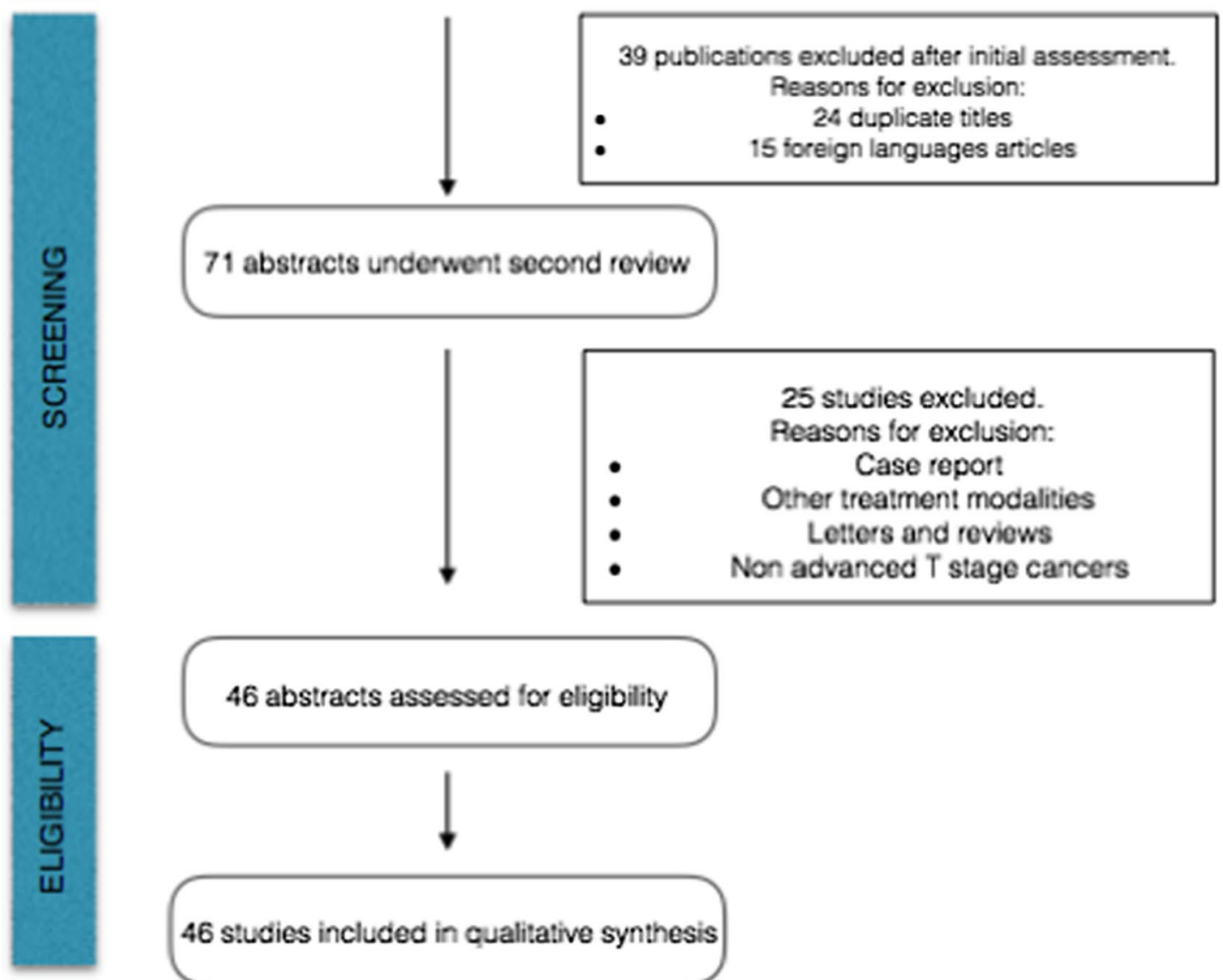

46 abstracts assessed for eligibility

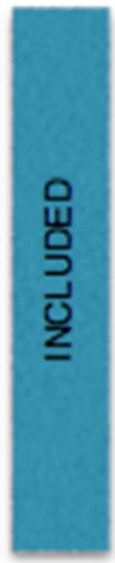

25 studies excluded.

Reasons for exclusion:

Not clearly describing aim/objective

- Not clearly reported inclusion and exclusion criteria

- Statistical data not clearly shown or detachable

Inadeguate local control data
- Focus on techniques and complications

21 studies included in qualitative synthesis (meta-analysis)

Fig. 1 This flow chart illustrates the process that was used to select articles for the review 
Table 2 Included studies with key results

\begin{tabular}{|c|c|c|c|c|c|c|c|c|}
\hline \multirow[t]{2}{*}{ Authors } & \multirow[t]{2}{*}{ QS* } & \multirow[t]{2}{*}{ Year } & \multirow[t]{2}{*}{ Pts n.** } & \multirow[t]{2}{*}{ Procedures } & \multicolumn{2}{|l|}{ Adjuvant radiotherapy (RT) } & \multirow[t]{2}{*}{ DFS (\%) } & \multirow[t]{2}{*}{ OS $(\%)$} \\
\hline & & & & & Primary & Neck & & \\
\hline Blanch [24] & 5 & 2011 & 26 & TLM & 1 & 6 & 80.40 & 71 \\
\hline Breda [25] & 5 & 2015 & 43 & TLM & 5 & 16 & 72.70 & 83.5 \\
\hline Bron [26] & 5 & 2005 & 16 & HPL & 3 & 7 & 83 & 80.26 \\
\hline Bussu [7] & 7 & 2013 & 43 & $\mathrm{CHP}$ & 15 patients (undetermined site) & & 70 & 68 \\
\hline Canis [27] & 5 & 2013 & 79 & TLM & 5 & 26 & 71.80 & 55.80 \\
\hline Canis [28] & 7 & 2013 & 159 & TLM & 55 & 84 & 81 & 59 \\
\hline Cho [29] & 4 & 2010 & 35 & SCPL (CHEP-CHP) THIP-THIEP & 0 & 7 & 82 & 78 \\
\hline Dufour [30] & 5 & 2004 & 118 & SCPL & 4 & 24 & 91.40 & 58 \\
\hline Gallo [31] & 6 & 2005 & 79 & CHP-CHEP & 10 & 0 & 85.60 & 75.30 \\
\hline Hinni [32] & 7 & 2007 & 106 & TLM & 15 & 29 & 52.54 & 49.80 \\
\hline Nakayama [33] & 3 & 2008 & 16 & SCL (CHEP-CHP) & 0 & 2 & 93.75 & 86 \\
\hline Pearson [34] & 5 & 2003 & 16 & TLM & 5 patients (undetermined site) & & 87.50 & 94.0 \\
\hline Peretti [35] & 5 & 2010 & 11 & TLM & 10 & 0 & 100 & 87.50 \\
\hline Peretti [36] & 6 & 2016 & 56 & TLM & 15 & 11 & 87 & 63.30 \\
\hline Qian [37] & 6 & 2014 & 20 & CPL & All of them underwent adjuvant RT & & 53 & 55.90 \\
\hline Rizzotto [38] & 6 & 2015 & 101 & THIP,THIEP & 24 & 7 & 65.90 & 77.70 \\
\hline Succo [39] & 6 & 2015 & 120 & THIEP+CAU, THIP+CAU & 28 & 13 & 91.70 & 63.81 \\
\hline Succo $[40]$ & 6 & 2016 & 555 & CHEP,CHP, THIP,THIEP & 72 & 123 & 92.80 & 84.60 \\
\hline Vilaseca [4] & 6 & 2010 & 147 & TLM & 25 & 37 & 70.20 & 53.10 \\
\hline Vilaseca [41] & 5 & 2016 & 154 & TLM & 46 & 58 & 67.60 & 55.60 \\
\hline Wilkie [42] & 5 & 2015 & 16 & TLM & 3 & 15 & 93.75 & 93.80 \\
\hline
\end{tabular}

$T L M$ transoral laser microsurgery, HPL horizontal partial laryngectomy, SCPL supracricohyoid partial laryngectomy, $C H E P$ cricohyoidoepiglottopexy, CHP cricohyoidopexy, STPL supratracheal partial laryngectomy, THIEP tracheohyoidoepiglottopexy, THIP tracheohyoidopexy, $C P L$ conservative partial laryngectomy, $C A U$ cricoarytenoid unit

*Quality score

**Patients number

a wide variety of treatment options are available and all of them provide similar survival outcomes, where considerations of patients' expectations and surgeons' skills play a fundamental role in decision making for advanced $\mathrm{T}$ stage laryngeal tumours. An analysis of the National Cancer Data Base (NCDB) information on laryngeal cancer has demonstrated a surprising decrease in 5-year overall survival for laryngeal cancer from 1985 to 2001, from 68.1 to $64.7 \%$, contrary to the most other malignancies [43].

In general, there has been a move from surgery towards chemoradiation therapy in the management of advanced laryngeal cancer patients, in attempt to preserve laryngeal functions; however, nonsurgical preservation approach do not offer a survival advantage compared to TL [14], and due to the evidence of objective failures of preservation laryngeal protocols [44-50], conservative surgery has been increasingly considered for selected tumours [51].

In the panorama of available surgical conservative techniques, both transoral and open conservative options, require specific expertise to ensure reproducible results.
TLM is known to be usually feasible for early glottic tumours, but there are evidences regarding the use of TLM for T3 glottic cancers with reported 5-year overall (53.1\%), disease-specific (70.2\%) and laryngectomy-free $(62.3 \%)$ survivals which make this technique indication still controversial [4, 52]. TLM has also demonstrated to be suitable for T3 supraglottic tumours with limited paraglottic or preepiglottic space invasion and also in some T4 cases with limited extension to tongue base or hypopharyngeal invasion. Accordingly, Hinni et al. [32] demonstrated a laryngeal preservation rate of $86 \%$ and a 5-year overall and diseasefree survival of 50 and 53\%, respectively; Rudert at el [53] confirmed a 5-year overall survival of $47 \%$ and Motta of $77 \%$ for $\mathrm{T} 3$ patients with a laryngeal preservation rate of $93.7 \%$ [54]. Vilaseca et al. [41] published one of the largest series on 154 patients with T3-T4 supraglottic cancers treated with TLM, by obtaining an OS and DFS rates ranging from 67 to $90 \%$, with a laryngeal preservation percentage up to 85 .

Literature concludes that TLM can be considered an established therapeutic option for selected advanced $\mathrm{T}$ stage 


\section{Overall survival}

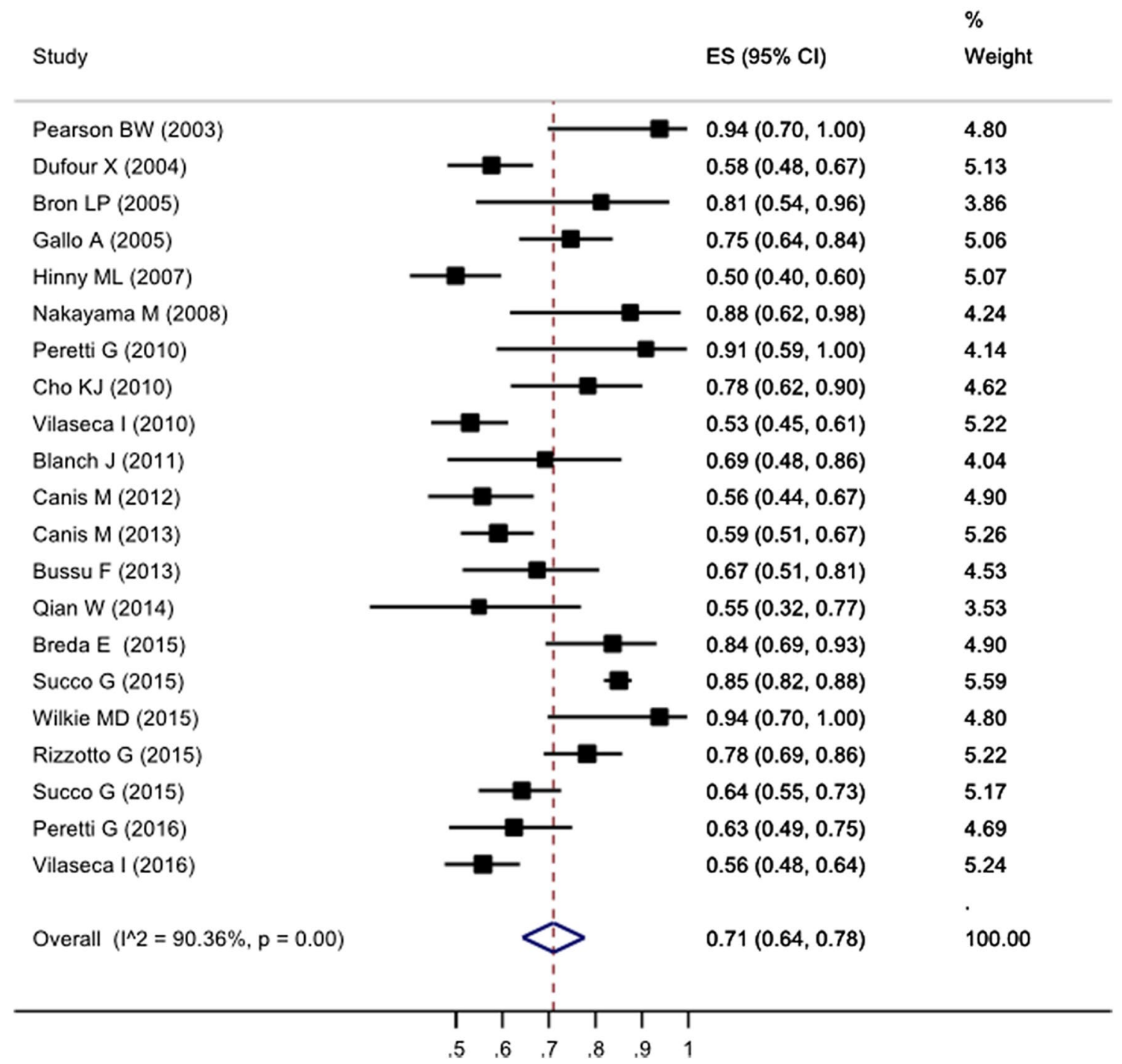

Fig. 2 Forest plot of overall survival (OS) for all patients, with pooled estimate and confidence intervals calculated using the DerSimonian Laird (random effects) model

laryngeal tumours, based on good oncologic, functional and economic considerations with an organ preservation rate over $60 \%$ in the vast majority of cases $[4,16,55,56]$.

In case of laryngeal tumours involving or originating from the anterior commissure (AC), difficulties in treatment could be related to tumour exposure and technical limitations of lack of perpendicular laser tool, which could be minimised by an enlarged resection and advanced surgical experience with TLM which allow the achievement of comparable oncologic and functional results with low morbidity $[24,34]$.

On the other hand, since Piquet and Chavalier [57] supracricoid laryngectomy has gained international acceptance, ensuring a reproducible and shared 5-year overall survival rate for all stages between 75 and 95\% [26, 29, 31, $33,40,58]$, together with a preservation successful rate in about $80 \%$ of cases $[59,60]$.

Given the lack of randomised control trials or even prospective studies in the topic of selection criteria for the use of different conservative surgical procedures as primary treatment for advanced laryngeal cancers, this study might offer a potential available evidence on the question. Despite several reports available in literature, all of them are mainly focused on one out of the several existing conservative techniques, whilst this study offers a systematic review of published articles and statistically robust results.

The finding of 79\% loco-regional control rate in over 1918 patients and about $80 \%$ of preservation rate gives credence 


\section{Disease free survival}

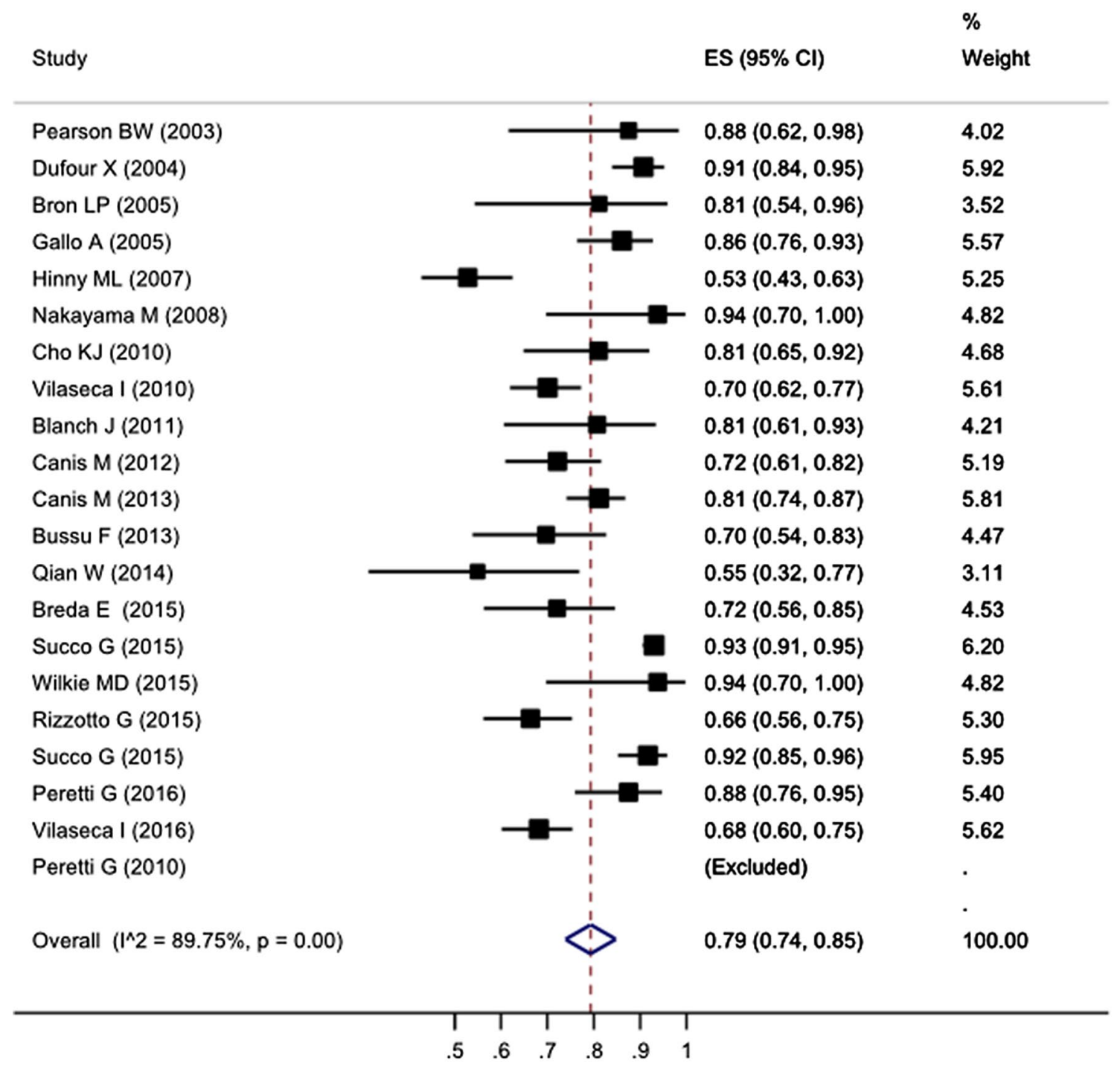

Fig. 3 Forest plot of disease-free survival (DFS) for all patients, with pooled estimate and confidence intervals calculated using the DerSimonian Laird (random effects) model

to the oncologic efficacy of these conservative surgical procedures. About $60 \%$ of patients were treated for advanced glottic cancers $(n=1134)$, whilst the remaining cases where advanced supraglottic tumours $(n=774)$. Although neck disease was not always clearly described and analysed, neck dissection was always recommended for supraglottic lesions and in case of clinical evidence of neck disease, but it was not possible to carry on statistical analysis due to lack of accurate data collection. It is obvious from our results that conservative surgical approach is a valid option for advanced (T3-T4) laryngeal lesions primary treatment, but due to the lower OS and DFS rate for the closed techniques (69 and $76 \%$ ) compared to open procedures ( 73 and $83 \%, p$ value not statistically significant), efforts should be made on individual patients' characteristics and expectations, and on available surgical skills and expertise for treatment.

Bussu et al. [7] in their series of patients with advanced laryngeal cancer, compared the oncological outcomes of 3 different therapeutic modalities [total laryngectomy, radiochemotherapy, or cricohyoidopexy (CHP)]. They observed a trend indicating better results in patients who underwent total laryngectomy vs those who underwent surgical and nonsurgical organ preservation strategies with respect to OS (90 vs 81 vs $68 \%$, respectively, at 2 years, $p=0.07$, Wilcoxon test) and DSS (92 vs 86 vs $72 \%$, respectively, at 2 years, $p=0.08$ ). In particular, among the cT4a cases, (2-year OS 93 vs 68\% for CHP and 54\% for nonsurgical treatment, $p=0.031)$ and for cT3 (2-year 


\section{Overall survival by technique}

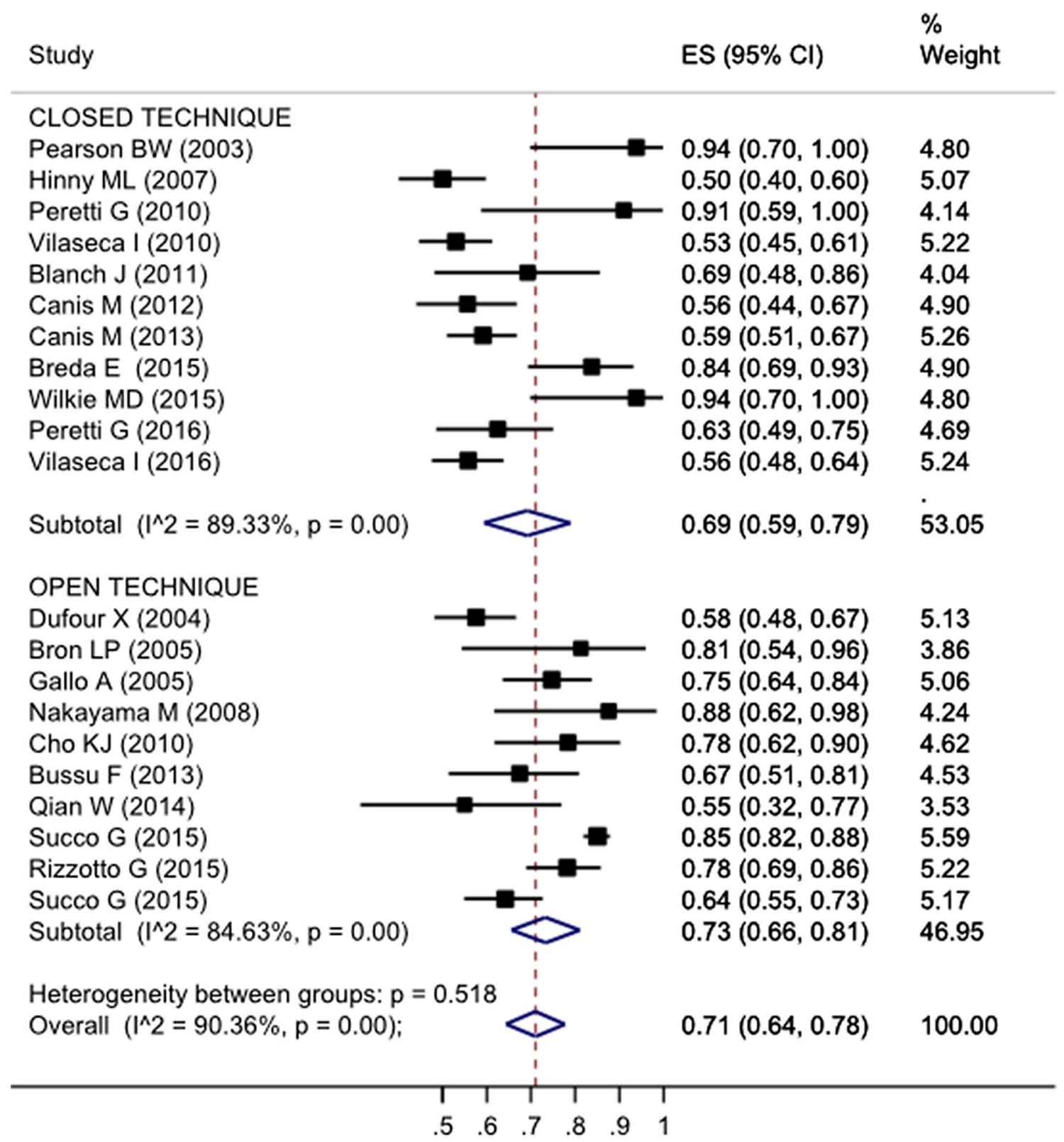

Fig. 4 Forest plot of overall survival (OS) sorted into two groups of treatment, with pooled estimate and confidence intervals calculated using the DerSimonian Laird (random effects) model

OS: $94 \%$ for total laryngectomy, $90 \%$ for CHP, $60 \%$ for radiochemotherapy, $p=0.0004$ ) a better OS and DSS were observed for patients treated by total laryngectomy. It is easy to understand that demolitive surgery provides better control of disease, although incurring a higher percentage of laryngeal function impairment and late sequelae that hamper patients' quality of life. The current trend for management of laryngeal cancer indicates that pursuing therapeutic options able to preserve laryngeal functionality provides the best quality of life for patients maintaining good oncological results.

In this perspective, post-operative irradiation has been reported to increase the rate of laryngeal preservation [35], accordingly to its previous mentioned indications. However, adjuvant RT is usually discouraged on the primary tumour site after partial laryngeal surgery because of its potential unfavourable functional late effects, including its rate in delaying patients recovery. In our meta-analysis, 


\section{Disease free survival by technique}

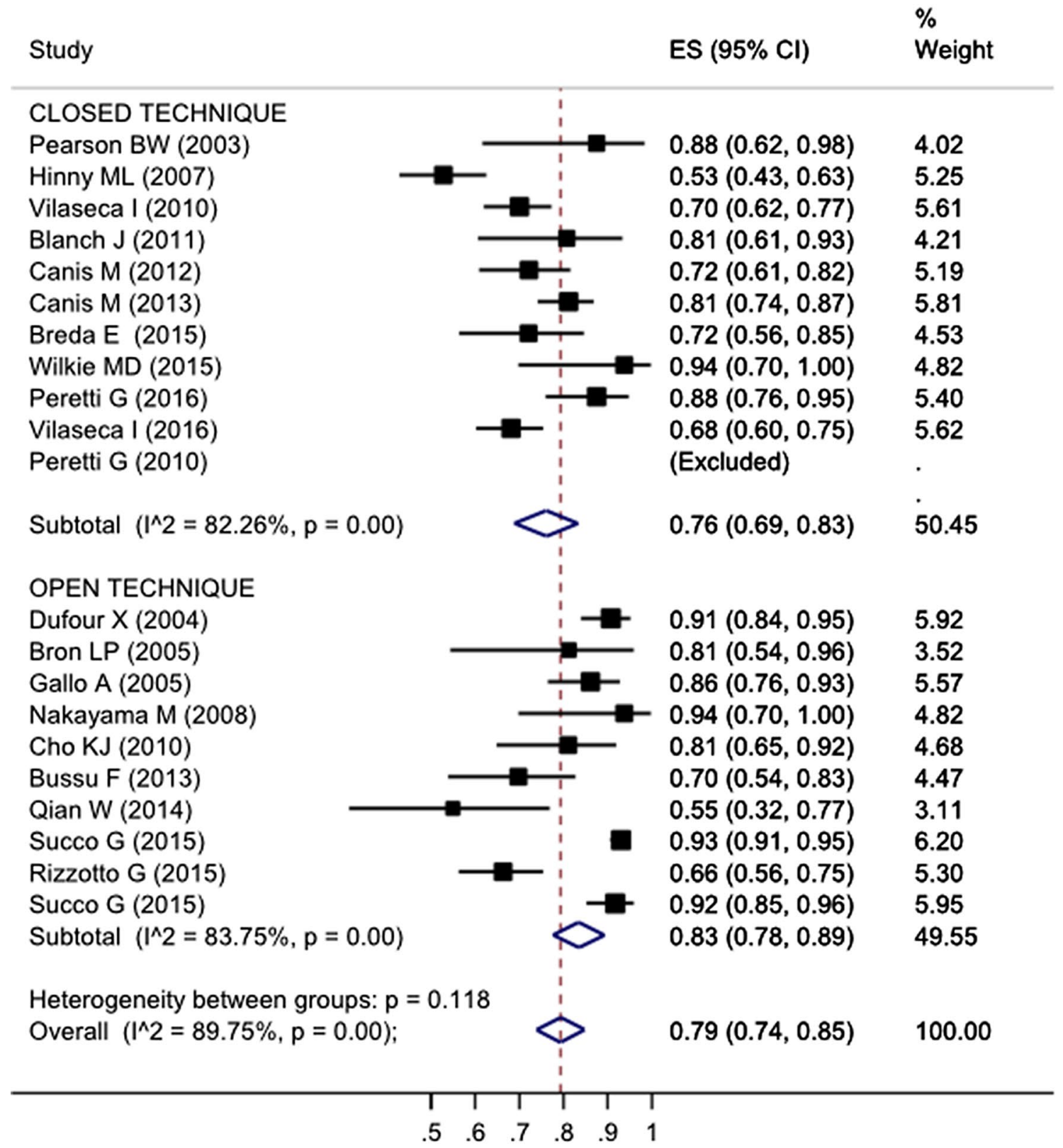

Fig. 5 Forest plot of disease-free survival (DFS) sorted into two groups of treatment, with pooled estimate and confidence intervals calculated using the DerSimonian Laird (random effects) model

crucial points were represented by positive resection margins. Closed techniques differed from the open ones in proposing a second surgical revisions in case of first pathological report indicative for presence of invasive cancers on resection margins; while, in the case of equivocal margins second procedures were not routinely performed. Only after a second pathological report indicative for deep positive margins, adjuvant RT was administered on the primary tumour site $[35,36,42]$. This is the reason why close cooperation with expert pathologists is of overwhelming importance. In accordance with these strict indications for adjuvant treatment, some authors such as Wilkie, Pearson and Peretti [34-36, 42] gained the highest rates of OS and DFS, in contrast to Hinni et al. [32], thus highlighting that TLM might reach excellent results when accurate selection of patients is made. Whilst, articles about closed technique showed less homogeneity in their outcome results, papers dealing with open procedures obtained more comparable 
data. This might be due to, not only to a longer surgical experience, but also to the presence of more consolidate indications for adjuvant radiotherapy, in comparison to those applied in TLM, so far, confirmed by a lower recurrence incidence rate $(p=0.118)$.

Although partial laryngectomies applicability to the management of locally advanced laryngeal carcinomas is gaining progressive consent among clinicians, upon our results, large T3 and more advanced pT4a tumours require several considerations to absolutely compare partial surgery radicality to that resulting from total laryngectomy procedure and primary (chemo)radiotherapy [27, 28, 39]. Pearson [34] has shown how the site of origin and $\mathrm{T}$ stage, despite they represent important considerations, do not represent overriding factors for TLM. Canis $[27,28]$ has demonstrated how TLM with/without adjuvant RT is a valid strategy for organ preservation and is an attractive therapeutic option even in locally advanced laryngeal cancers. Accordingly, open access supports en bloc tumour removal but, arytenoids cartilage fixation, posterior commissure invasion, cricoid cartilage invasion and extra-laryngeal cancer spread do still represent major contraindications for partial procedure indications [33].

A very important secondary endpoint is the functional outcome, however the data was not always specified in the mentioned papers making impossible any comparative analysis among TLM and OPL. In fact, not every patient treated conservatively by both TLM or OPL have a complete restoration of laryngeal function, even if this represent a goal reached by the great majority of them.

\section{Conclusion}

The aim of this review was to show that evidence for surgical conservative strategies still remain low, despite the encouraging results over the last fifteen years. Future perspective randomised trials with comparable cohorts should aim to improve our levels of evidence for treatment decision making.

\section{Compliance with ethical standards}

Conflict of interst Authors declares that they have no conflict of interest.

Research involving human participants and/or animals All procedures performed in studies involving human participants were in accordance with the ethical standards of the institutional and/or national research committee and with the 1964 Helsinki Declaration and its later amendments or comparable ethical standards.

Informed consent Informed consent was obtained from all individual participants included in the study.

\section{References}

1. American Cancer Society. Cancer facts figs. 2010. Atlanta, GA: American Cancer Society; 2010

2. Edge SB, Byrd DR, Compton CC, Fritz AG, Greene FL, Trotti A III (eds) (2010) Larynx AJCC cancer staging manual, 7th edn. Springer, New York, pp 57-62

3. Canis M, Martin A, Ihler F et al (2014) Transoral laser microsurgery in treatment of pT2 and pT3 glottic laryngeal squamous cell carcinoma-results of 391 patients. Head Neck 36:859-866

4. Vilaseca I, Bernal-Sprekelsen M, Luis Blanch J (2010) Transoral laser microsurgery for T3 laryngeal tumors: prognostic factors. Head Neck 32:929-938

5. Thomas L, Drinna M, Natesh B, Mehanna H, Jones T, Paleri V (2012) Open conservation partial laryngectomy for laryngeal cancer: a systematic review of English language literature. Can Treat Rev 38:203-2011

6. Van Luschka H (1971;) Der Kehlkopf des Menschen. Laupp, Tubingen

7. Bussu F, Paludetti G, Almadori G et al (2013) Comparison of total laryngectomy with surgical (cricohyoidopexy) and nonsurgical organ-preservation modalities in advanced laryngeal squamous cell carcinomas: a multicenter retrospective analysis. Head Neck 35:554-561

8. O'Neill CB, O'Neill JP, Atoria CL et al (2014) Treatment complications and survival in advanced laryngeal cancer: a population based analysis. Laryngoscope 124:2707-2713

9. Labayle J, Bismuth R total laryngectomy with reconstitution. Ann Otolaryngol Chir Cervicofac, 1971

10. Piquet JJ, Desaulty A, Decroix G (1974) Crico-hyoido-epiglottopexy. Surgical technic and functional results. Ann Otolaryngol Chir Cervicofac 91:681-686

11. Steiner W (1988) Experience in endoscopic laser surgery of malignant tumors of the upper aero-digestive tract. Adv Otorhinolaryngol 39:135-144

12. Licitra L, Bernier J, Grandi C et al (2003) Cancer of the larynx. Brit Rev Oncol Hematol 47:65-80

13. Forastiere AA, Goepfert H, Maor M et al (2003) Concurrent chemotherapy and radiotherapy for organ preservation in advanced laryngeal cancer. N Eng J Med 349:2091-2098

14. Pfister DG, Laurie SA, Mendenhall WM et al (2006) American Society of Clinical Oncology Clinical Practice Guidelines for the use of larynx-preservation strategies in the treatment of laryngeal cancer. J Clin Oncol 22:1-12

15. Gallo A, de Vincentiis M, Manciocco V, Simonelli M, Fiorella ML, Shah JP (2002) CO2 laser cordectomy for early-stage glottic carcinoma: a long-term follow-up of 156 cases. Laryngoscope 112:370-374

16. Steiner W (1993) Results of curative laser microsurgery of laryngeal carcinomas. Am J Otolaryngol 14:116-121

17. Pellini R, Pichi B, Ruscito P et al (2008) Supracricoid partial laryngectomies after radiation failure: a multi-institutional series. Head Neck 30:372-379

18. Piazza C, Peretti G, Cattaneo A, Garrubba F, De Zinis LO, Nicolai P (2007) Salvage surgery after radiotherapy for laryngeal cancer: from endoscopic resections to open-neck partial and total laryngectomies. Arch Otolaryngol Head Neck Surg 133:1037-1043

19. Shamseer L, Moher D, Clarke M, Ghersi D, Liberati A et al (2014) Preferred reporting items for systematc review and meta-analysis protocols (PRISMA-P) 2015: elaboration and explanation. BMJ 349: 97647

20. Sedgwick P (2015) Meta-analysis: testing for reporting bias. BMJ 350:g7858

21. Goodman S, Dickersin K (2011) Metabias: a challenge for comparative effectiveness research. Ann Intern Med;155:61-62 
22. Higgins JP, Thompson SG, Deeks JJ, Altman DG (2003) Measuring inconsistency in meta-analysis. BMJ 327:557-560

23. Sedgwick P. Meta-analysis: what is heterogeneity? BMJ 2015; 350:h1435

24. Blanch JL, Vilaseca I, Caballero M, Moragas M, Berenguer J, Bernal-Sprekelsen M (2011) Outcome of transoral laser microsurgery for T2-T3 tumors growing in the laryngeal anterior commissure. Head Neck 33:1252-1259

25. Breda E, Catarino R, Monteiro E (2015) Transoral laser microsurgery for laryngeal carcinoma: Survival analysis in a hospital-based population. Head Neck 37:1181-1186

26. Bron L, Brossard E, Monnier P, Pasche P (2000) Supracricoid partial laryngectomy with cricohyiodoepiglottopexy for glottic and supraglottic carcionma. Laryngoscope 110:627-634

27. Canis M, Ihler F, Martin A, Wolff HA, Matthias C, Steiner W (2013) Organ preservation in T4a laryngeal cancer: is transoral laser microsurgery an option? Our Arch Otorhinolaryngol 270:2719-2727

28. Canis M, Martin A, Ihler F et al (2013) Results of transoral laser microsurgery for supraglottic carcinoma in 277 patients. Our Arch Otorhinolaryngol 270:2315-2326

29. Farrag TY, Koch WM, Cummings CW et al (2007) Supracricoid laryngectomy outcomes: the Johns Hopkins experience. Laryngoscope 117:129-132

29. Cho KJ, Too YH, Sun DI, Kim MS (2010) Supracricoid laryngectomy: oncologic validity and functional safety. Our Arch Otorhinolaryngol 267:1919-1925

30. Dufour X, Hans S, De Mones E, Brasnu D, Ménard M, Laccourreye O (2004) Local control after supracricoid partial laryngectomy for "advanced" endolaryngeal squamous cell carcinoma classified as T3. Arch Otolaryngol Head Neck Sure 130:1092-1099

31. Gallo A, Manciocco V, Simonelli M, Pagliuca G, D'Arcangelo E, de Vincentiis M (2005) Supracricoid partial laryngectomy in the treatment of laryngeal cancer. Univariate and multivariate analysis of prognostic factors. Arch Otolaryngol Head Neck Surg 131:620-625

32. Hinni ML, Salassa JR, Grant DG et al (2007) Transoral laser microsurgery for advanced laryngeal cancer. Arch Otolaryngol Head Neck Surg 133:1198-1204

33. Nakayama M, Okatmoto M, Minamoto S et al (2008) Supracricoid laryngectomy with cricohyiodoepiglotto-pexy of cricohyoidopexy: experience on 32 patients. Auris Nasus Larynx 35:77-82

34. Pearson BW, Salassa JR (2003) Transoral laser microresection for cancer of the larynx involving the anterior commissure. Laryngoscope 113:1104-1112

35. Peretti G, Piazza C, Ansarin M et al (2010) Transoral CO2 laser microsurgery for Tis-T3 supraglottic squamous cell carcinomas. Our Arch Otorhinolaryngol 267:1735-1742

36. Peretti G, Piazza C, Penco S et al (2016) Transoral laser microsurgery as primary treatment for selected $\mathrm{T} 3$ glottic and supraglottic cancers. Head Neck 38:1107-1112

37. Qian W, Zhu G, Wang Y et al (2014) Multi-modality management for loco-regionally advanced laryngeal and hypopharyngeal cancer: balancing the benefit of efficacy and functional preservation. Med Once 31:178

38. Rizzotto G, Crosetti E, Lucioni M et al (2015) Oncologic outcomes of supratracheal laryngectomy: critical analysis. Head Neck 37:1417-1424

39. Succo G, Bussi M, Presutti L et al (2015) Supratracheal laryngectomy: current indications and contraindications. Acta Otorhinolaryngol Ital 35:146-156

40. Succo G, Corsetti E, Berlin A et al (2016) Benefits and drawbacks of open partial horizontal laryngectomies, Part B: Intermediate and selected advanced stage laryngeal carcinoma. Head Neck 38:649-657
41. Vilaseca I, Blanch JL, Berenguer J et al (2016) Transoral laser microsurgery for locally advanced (T3-T4a) supraglottic squamous cell carcinoma: Sixteen years of experience. Head Neck 38:1050-1057

42. Wilkie MD, Lightbody KA, Lythgoe D, Tandon S, Lancaster J, Jones TM (2015) Transoral laser microsurgery for early and moderately advanced laryngeal cancers: outcomes from a single centralized United Kingdom centre. Our Arch Otorhinolaryngol 272:695-704

43. Hoffman HT, Porter K, Karnell LH et al (2006) Laryngeal cancer in the United States: changes in demographics, patterns of care, and survival. Laryngoscope 116(Suppl 111):1-13

44. Holsinger FC (2008) Swing of the pendulum: optimizing functional outcomes in larynx cancer. Curr Oncol Rep 10:170-175

45. Kotz T, Costello R, Li Y, Posner MR (2004) Swallowing dysfunction after chemoradiation for advanced squamous cell carcinoma of the head and neck. Head Neck 26:365-372

46. Rosenthal DI, Lewin JS, Eisbruch A (2006) Prevention and treatment of dysphagia and aspiration after chemoradiation for head and neck cancer. J Clin Oncol 24:2636-2643

47. Pfister DG, Laurie SA, Weinstein GS et al (2006) American Society of Clinical Oncology clinical practice guideline for the use of larynx-preservation strategies in the treatment of laryngeal cancer. J Clin Oncol 24:3693-3704

48. Roh JL, Kim AY, Cho MJ (2005) Xerostomia following radiotherapy of the head and neck affects vocal function. J Clin Oncol 23:3016-3023

49. Campbell BH, Spinelli K, Marbella AM, Myers KB, Kuhn JC, Layde PM (2004) Aspiration, weight loss, and quality of life in head and neck cancer survivors. Arch Otolaryngol Head Neck Surg 130:1100-1103

49. Hartl DM, Ferlito A, Brasnu DF, Langendijk JA, Rinaldo A, Silver CE, Wolf GT (2011) Evidence-based review of treatment options for patients with glottic cancer. Head Neck 22:1638-1648

50. Rademaker AW, Vonesh EF, Logemann JA et al (2003) Eating ability in head and neck cancer patients after treatment with chemoradiation: a 12-month follow-up study accounting for dropout. Head Neck 25:1034-1041

51. Suarez C, Rodrigo JP, Silver CE et al (2012) Laser surgery for early to moderate advanced glottic, supraglottic, and hypo pharyngeal cancers. Head Neck 34:1028-1035

53. Rudert HH, Werner JA, Hoft S (1999) Transoral carbon dioxide laser resection of supraglottic carcinoma. Ann Otol Rhinol Laryngal 108:819-827

54. Motta G, Esposito E, Testa D, Iovine R, Motta S (2004) CO2 laser treatment supraglottic cancer. Head Neck 26:442-446

55. Peretti G, Piazza C, Cocco D et al (2010) Transoral CO2 laser treatment for Tis-T3 glottic cancer: the University of Brescia experience on 595 patients. Head Neck 32:977-983

56. Preuss SF, Cramer K, Klussmann JP, Eckel HE, Guntinas-Lichius O (2009) Transoral laser surgery for laryngeal cancer: outcome, complications and progsnotic factors in 275 patients. EJSO 35:235-240

57. Piquet J, Chevalier D (1991) Subtotal laryngectomy with cricohioido-epiglotto-pexy for the treatment of extended glottic carcinomas. Am J Surg 162:357-361

59. Lima RA, Freitas EQ, Dias FL, et al. Supracricoid laryngectomy with cricohyiodoepiglottopexy for advanced glottic cancer. Head Neck 2006;28:481-486

60. de Vincentiis M, Minni A, Gallo A, Di Nardo A (1998) Supracricoid partial laryngectomies: oncologic and functional results. Head Neck 20:504-509 